doi:10.13108/2015-7-4-15

UDC 517.53

\title{
EXACTNESS OF ESTIMATES FOR $k$-th ORDER OF DIRICHLET SERIES IN A SEMI-STRIP
}

\author{
N.N. AITKUZHINA, A.M. GAISIN
}

Dedicated to the memory of professor Igor' Fedorovich Krasichkov-Ternovskii

\begin{abstract}
We study Dirichlet series converging only in a half-plane such that their sequence of exponents admits an extension to a "regular" sequence. We prove the exactness of two-sided estimates for $k$-order of the sum of the Dirichlet series in a semi-strip whose width depends on the special distribution density of the exponents.
\end{abstract}

Keywords: $k$-order of the Dirichlet series in a semi-strip, entire functions with a prescribed asymptotics on the positive axis.

Mathematics Subject Classification: 3010

Let $\Lambda=\left\{\lambda_{n}\right\}\left(0<\lambda_{n} \uparrow \infty\right)$ be a sequence satisfying the condition

$$
\varlimsup_{n \rightarrow \infty} \frac{\ln n}{\lambda_{n}}=H<\infty .
$$

In studying entire functions

$$
F(s)=\sum_{n=1}^{\infty} a_{n} e^{\lambda_{n} s} \quad(s=\sigma+i t)
$$

defined by everywhere convergent Dirichlet series, Ritt introduced the notion of $R$-order [1]:

$$
\rho_{R}=\varlimsup_{\sigma \rightarrow+\infty} \frac{\ln \ln M(\sigma)}{\sigma},
$$

where $M(\sigma)=\sup _{|t|<\infty}|F(\sigma+i t)|$. We note that by condition (1) series (2) converges absolutely in the whole plane. It is known that $\ln M(\sigma)$ is an increasing convex function of $\sigma, \lim _{\sigma \rightarrow+\infty} \ln M(\sigma)=$ $+\infty$. The quantity

$$
\rho_{s}=\varlimsup_{\sigma \rightarrow+\infty} \frac{\ln ^{+} \ln M_{s}(\sigma)}{\sigma} \quad\left(a^{+}=\max (a, 0)\right)
$$

is called $R$-order of function $F$ in strip $S\left(a, t_{0}\right)=\left\{s=\sigma+i t:\left|t-t_{0}\right| \leqslant a\right\}$. Here $M_{s}(\sigma)=$ $\max _{\left|t-t_{0}\right| \leqslant a}|F(\sigma+i t)|$.

In [2] sufficient conditions for $\Lambda$ and $a$ ensuring $\rho_{R}=\rho_{S}$ were obtained. The most general results on relation between $\rho_{R}$ and $\rho_{s}$ was established by A.F. Leontiev [3].

Similar issues in the case when $H=0$ and the convergence domain of series $(2)$ is the half-plane $\Pi_{0}=\{s=\sigma+i t: \sigma<0\}$ were studied by A.M. Gaisin in [4].

N.N. Aitkuzhina, A.M. Gaisin, Exactness of estimates for $k$-Th order of Dirichlet Series in A SEMI-STRIP.

(c) Aitkuzhina N.N., Gaisin A.M. 2015.

The work is supported by RFBR (grant no. 15-01-01661) and the Program of fundamental research of the Department of Mathematics of RAS "Modern problems of theoretical mathematics", project "Complex analysis and functional equations".

Submitted October 6, 2015. 
As $H=0$, if series (2) converges in half-plane $\Pi_{0}$, it converges absolutely in $\Pi_{0}$. Then the sum of series $F$ is analytic in this half-plane. The class of all unbounded analytic functions represented by Dirichlet series (2) converging just in half-plane $\Pi_{0}$ is denoted by $D_{0}(\Lambda)$.

Let $S\left(a, t_{0}\right)=\left\{s=\sigma+i t:\left|t-t_{0}\right| \leqslant a, \sigma<0\right\}$ be a semi-strip. The quantities

$$
\rho_{R}=\varlimsup_{\sigma \rightarrow 0-} \frac{\ln ^{+} \ln M(\sigma)}{|\sigma|^{-1}}, \quad \rho_{s}=\varlimsup_{\sigma \rightarrow 0-} \frac{\ln ^{+} \ln M_{s}(\sigma)}{|\sigma|^{-1}}
$$

are called Ritt orders of function $F$ in half-plane $\Pi_{0}$ and semi-strip $S\left(a, t_{0}\right)$ [4]. In what follows we call $\rho_{R}$ and $\rho_{s}$ orders in the half-plane and the semi-strip. If it is necessary, instead of $\rho_{R}$ and $\rho_{s}$ we shall write $\rho_{R}(F)$ and $\rho_{s}(F)$.

It was shown in [4] that condition

$$
\lim _{n \rightarrow \infty} \frac{\ln \lambda_{n}}{\lambda_{n}} \ln n=0
$$

is sufficient for order $\rho_{R}$ of each function $F \in D_{0}(\Lambda)$ to be

$$
\rho_{R}=\varlimsup_{n \rightarrow \infty} \frac{\ln \lambda_{n}}{\lambda_{n}} \ln ^{+}\left|a_{n}\right| .
$$

Let sequence $\Lambda$ have a finite upper density $D$. Then

$$
L(z)=\prod_{n=1}^{\infty}\left(1-\frac{z^{2}}{\lambda_{n}^{2}}\right) \quad(z=x+i y)
$$

is an entire function of exponential type. If $h(\varphi)$ is the growth indicatri, and $\tau$ is a type of function $L$, then $\tau=h\left({ }_{-}^{+} \frac{\pi}{2}\right) \leqslant \pi D^{*}$ ( $D^{*}$ is the averaged upper density of sequence $\Lambda$ ) [2]. Assume that

$$
|L(x)| \leqslant e^{g(x)} \quad(x \geqslant 0), \quad \lim _{x \rightarrow+\infty} \frac{g(x) \ln x}{x}=0,
$$

where $g$ is a non-negative on $\mathbb{R}_{+}=[0, \infty)$ function. In this case the adjoint diagram of function $L$ is the segment $I=[-\tau i, \tau i], h(\varphi)=\tau|\sin \varphi|$.

In [4] the following theorem was proved.

Theorem I. Suppose that function L satisfies conditions (4) and has type $\tau(0 \leqslant \tau<\infty)$. We let $q=q(L)$, where

$$
q(L)=\varlimsup_{n \rightarrow \infty} \frac{\ln \lambda_{n}}{\lambda_{n}} \ln \left|\frac{1}{L^{\prime}\left(\lambda_{n}\right)}\right| .
$$

Then order $\rho_{s}$ in the half-plane $S\left(a, t_{0}\right)$ as $a>\tau$ and order $\rho_{R}$ of each function $F \in D_{0}(\Lambda)$ in half-plane $\Pi_{0}$ satisfy the eistimates

$$
\rho_{s} \leqslant \rho_{R} \leqslant \rho_{s}+q .
$$

The left estimate in (6) is exact [4]. In the general situation the right estimate is not exact, moreover, the pair of conditions (4) can fail. But there can exist an entire function $Q$ of exponential type with simple zeroes at the points of sequence $\Lambda$, for which conditions (4) hold and $q(Q)=q^{*}$, where

$$
q^{*}=\varlimsup_{n \rightarrow \infty} \frac{\ln \lambda_{n}}{\lambda_{n}} \int_{0}^{1} \frac{n\left(\lambda_{n} ; t\right)}{t} d t,
$$

$q(Q)$ is the quantity defined in the same way as $q(L)$ in $(5)$, and $n\left(\lambda_{n} ; t\right)$ is the amount of points $\lambda_{k} \neq \lambda_{n}$ in the segment $\left\{x:\left|x-\lambda_{n}\right| \leqslant t\right\}$. Paper [5] is devoted to constructing such entire functions $Q$ with a prescribed subset of zeroes $\Lambda$ and a prescribed asymptotics at the real axis. It turns out that in terms of a special distribution density $G(R)$ of sequence $\Lambda$ one can provide conditions under which the estimates

$$
\rho_{s} \leqslant \rho_{R} \leqslant \rho_{s}+q^{*}
$$


hold true, where $\rho_{s}$ is the order in a semi-strip $S\left(a, t_{0}\right)$ of the width greater than $\left.2 \pi G(R)\right)$ and these estimates are the best possible in class $D_{0}(\Lambda)$ [6]. Similar estimates for $k$-orders were obtained in [7]. The aim of the paper is prove the exactness of these estimates.

\section{DEFINITIONS AND NEEDED FACTS}

Let $\Lambda=\left\{\lambda_{n}\right\},\left(0<\lambda_{n} \uparrow \infty\right)$ be a sequence having a finite upper density, $L$ be the class of positive continuous and unboundedly increasing on $[0, \infty)$ functions. By $K$ we denote a subclass of functions $h$ in $L$ such that $h(0)=0, h(t)=o(t)$ as $t \rightarrow \infty, \frac{h(t)}{t} \downarrow$ as $t \uparrow\left(\frac{h(t)}{t}\right.$ decreases monotonically as $t>0)$. In particular, if $h \in K$, the $h(2 t) \leqslant 2 h(t)(t>0), h(t) \leqslant h(1) t$ as $t \geqslant 1$.

$K$-density of sequence $\Lambda$ is the quantity

$$
G(K)=\inf _{h \in K} \varlimsup_{t \rightarrow \infty} \frac{\mu_{\Lambda}(\omega(t))}{h(t)}
$$

where $\omega(t)=[t, t+h(t))$ is a semi-interval, $\mu_{\Lambda}(\omega(t))$ is the amount of points of $\Lambda$ lying in semi-interval $\omega(t)$.

Let $\Omega=\{\omega\}$ be a family of semi-intervals $\omega=[a, b)$. By $|\omega|$ we denote the length of $\omega$. Each sequence $\Lambda=\left\{\lambda_{n}\right\},\left(0<\lambda_{n} \uparrow \infty\right)$ generates an integer-valued counting measure $\mu_{\Lambda}$ :

$$
\mu_{\Lambda}(\omega)=\sum_{\lambda_{n} \in \omega} 1, \quad \omega \in \Omega
$$

Let $\mu_{\Gamma}$ be a counting measure generated by the sequence $\Gamma=\left\{\mu_{n}\right\},\left(0<\mu_{n} \uparrow \infty\right)$. Then the inclusion $\Lambda \subset \Gamma$ means that $\mu_{\Lambda}(\omega) \leqslant \mu_{\Gamma}(\omega)$ for each $\omega \in \Omega$. In this case we shall say that measure $\mu_{\Gamma}$ majorizes measure $\mu_{\Lambda}$.

By $D(K)$ we denote the infimum of numbers $b(0 \leqslant b<\infty)$ for which there exists measure $\mu_{\Gamma}$ majorizing $\mu_{\Lambda}$ such that

$$
|M(t)-b t| \leqslant h(t) \quad(t \geqslant 0)
$$

for some function $h \in K$. Here $\Lambda=\left\{\lambda_{n}\right\}, \Gamma=\left\{\mu_{n}\right\}, M(t)=\sum_{\mu_{n} \leqslant t} 1$.

It was shown in $[6]$ that $D(K)=G(K)$.

The quantity

$$
\rho_{k}=\varlimsup_{\sigma \rightarrow 0_{-}} \frac{\ln _{k} M(\sigma)}{|\sigma|^{-1}} \quad(k \geqslant 2)
$$

is called $k$-order of function $F \in D_{0}(\Lambda)$ in half-plane $\Pi_{0}=\{s: \sigma=\operatorname{Re} s<0\}$ [7]. Here $\ln _{0} t=t, \ln _{k} t=\underbrace{\ln \ln \ldots \ln t}_{k}(k \geqslant 1)$. In view of the definition of $k$-order $(9)$ we see that $\rho_{2}=\rho_{R}$, where $\rho_{R}$ is the $R$-order in half-plane $\Pi_{0}[4]$.

The following theorem was proven in [7].

Theorem II. The condition

$$
\lim _{n \rightarrow \infty} \frac{\ln n \ln _{k-1} \lambda_{n}}{\lambda_{n}}=0 \quad(k \geqslant 2)
$$

is necessary and sufficient for $k$-order $\rho_{k}$ of each function $F \in D_{0}(\Lambda)$ to satisfy the formula

$$
\rho_{k}=\varlimsup_{n \rightarrow \infty} \frac{\ln \left|a_{n}\right|}{\lambda_{n}} \ln _{k-1} \lambda_{n} \quad\left(k \geqslant 2 ; 0 \leqslant \rho_{R} \leqslant \infty\right) .
$$

We observe that formula (3) is a particular case of identity (11).

In the same way one introduces the notion of $k$-order $\rho_{s}^{(k)}$ in semi-strip $S\left(a, t_{0}\right)$. For the sake of convenience, we shall still denote it by $\rho_{s}$. 
We introduce the following classes of functions:

$$
\begin{aligned}
& L_{k}=\left\{h \in L: h(x) \ln _{k-1} x=o(x), \quad x \rightarrow \infty\right\} \quad(k \geqslant 2), \\
& S=\left\{h \in K: \quad d(h)=\varlimsup_{x \rightarrow \infty} \frac{h(x) \ln h(x)}{x \ln \frac{x}{h(x)}}<\infty\right\}, \\
& R_{k}=\left\{h \in S: h(x) \ln \frac{x}{h(x)}=o\left(\frac{x}{\ln _{k-1} x}\right), \quad x \rightarrow \infty\right\} \quad(k \geqslant 2) .
\end{aligned}
$$

The following theorem was proved in paper [7].

Theorem III. Let $\Lambda=\left\{\lambda_{n}\right\},\left(0<\lambda_{n} \uparrow \infty\right)$ be a sequence satisfying the conditions

1) $\Lambda(x+\rho)-\Lambda(x) \leqslant c \rho+d+\frac{\varphi(x)}{\ln ^{+} \rho+1},(\rho \geqslant 0)$, where $\Lambda(x)=\sum_{\lambda_{n} \leqslant x} 1$, $\varphi$ is a function in $L_{k}$ $(k \geqslant 2)$;

2) $q_{k}^{*}=\varlimsup_{n \rightarrow \infty} \frac{\ln _{k-1} \lambda_{n}}{\lambda_{n}} \int_{0}^{1} \frac{n\left(\lambda_{n} ; t\right)}{t} d t<\infty(k \geqslant 2)$, where $n\left(\lambda_{n} ; t\right)$ is the amount of points of $\lambda_{k} \neq \lambda_{n}$ lying in the segment $\left\{x:\left|x-\lambda_{n}\right| \leqslant t\right\}$.

If density $R_{k}$ of sequence $\Lambda$ is equal to $G(R)$, then $k$-order $\rho_{s}$ of each function $F \in D_{0}(\Lambda)$ in semi-strip $S\left(a, t_{0}\right)$ as $a>\pi G\left(R_{k}\right)$ and order $\rho_{R}$ of this function in half-plane $\Pi_{0}$ satisfies the estimates

$$
\rho_{s} \leqslant \rho_{k} \leqslant \rho_{s}+q_{k}^{*} \quad(k \geqslant 2) .
$$

As it is known, estimate $\rho_{s} \leqslant \rho_{k}$ in (12) is exact. In what follows we discuss the exactness of inequality $\rho_{k} \leqslant \rho_{s}+q_{k}^{*}(k \geqslant 2)$.

\section{MAIN THEOREM ON EXACT ESTIMATES FOR $k$-ORDER}

The main result of the paper is the following theorem.

Theorem 1. Let $\Lambda$ be a sequence satisfying the assumptions of Theorem III. Then there exists a function $F \in D_{0}(\Lambda)$ such that $\rho_{k}(F)=\rho_{s}(F)+q^{*}$, where $\rho_{k}(F)$ is the order in halfplane $\Pi_{0}$, and $\rho_{s}(F)$ is order in semi-strip $S\left(a, t_{0}\right),(a>\pi G(R))$.

Corollary. Suppose that $\Lambda$ satisfies the assumptions of Theorem 1. Order $\rho_{k}(F)$ of each function $F \in D_{0}(\Lambda)$ is equal to order $\rho_{s}(F)$ in each semi-strip $S\left(a, t_{0}\right)(a>\pi G(R))$ if and only $q^{*}=0$.

In the proof of Theorem 1 we shall make use of

Theorem IV [6]. Let $\Lambda=\left\{\lambda_{n}\right\}\left(0<\lambda_{n} \uparrow \infty\right)$ be a sequence having a finite $S$-density $G(S)$. Then for each $b>G(S)$ there exists a sequence $\Gamma=\left\{\mu_{n}\right\} \quad\left(0<\mu_{n} \uparrow \infty\right)$ containing $\Lambda$ and having density $b$ such that the entire function

$$
Q(z)=\prod_{n=1}^{\infty}\left(1-\frac{z^{2}}{\mu_{n}^{2}}\right) \quad(z=x+i y)
$$

of exponential type $\pi b$ possesses the properties:

1) $Q\left(\lambda_{n}\right)=0, Q^{\prime}\left(\lambda_{n}\right) \neq 0$ for each $\lambda_{n} \in \Lambda$;

2) there exists $H \in S$ such that

$$
\ln |Q(x)| \leqslant A H(x) \ln ^{+} \frac{x}{H(x)}+B ;
$$

3) if $\Lambda(x)=\sum_{\lambda_{n} \leqslant x} 1$, and

$$
\Lambda(x+\rho)-\Lambda(x) \leqslant a \rho+b+\frac{\varphi(x)}{\ln ^{+} \rho+1} \quad(\rho \geqslant 0)
$$


( $\varphi$ is an arbitrary non-negative non-decreasing function defined on the ray $[0, \infty)$, $\left.1 \leqslant \varphi(x) \leqslant \alpha x \ln ^{+} x+\beta\right)$, then there exists a sequence $\left\{r_{n}\right\}, 0<r_{n} \uparrow \infty, r_{n+1}-r_{n}=O\left(H\left(r_{n}\right)\right)$ as $n \rightarrow \infty$, such that for $x=r_{n}(n \geqslant 1)$

$$
\ln |Q(x)| \geqslant-C H(x) \ln ^{+} \frac{x}{H(x)}-2 \varphi(x)-D
$$

4) if

$$
\Delta=\varlimsup_{n \rightarrow \infty} \frac{1}{\lambda_{n}} \int_{0}^{1} \frac{n\left(\lambda_{n} ; t\right)}{t} d t<\infty
$$

then under condition (14)

$$
|\ln | \frac{1}{Q^{\prime}\left(\lambda_{n}\right)}\left|-\int_{0}^{1} \frac{n\left(\lambda_{n} ; t\right)}{t} d t\right| \leqslant E H\left(\lambda_{n}\right) \ln ^{+} \frac{\lambda_{n}}{H\left(\lambda_{n}\right)}+2 \varphi\left(\lambda_{n}\right)+F \ln \lambda_{n}+L \quad(n \geqslant 1),
$$

where $n\left(\lambda_{n} ; t\right)$ is the amount of points $\lambda_{k} \neq \lambda_{n}$ in the segment $\left\{x:\left|x-\lambda_{n}\right| \leqslant t\right\}$.

Here all constants are finite and positive.

Let $\Lambda=\left\{\lambda_{n}\right\}$ be a sequence satisfying assumptions of Theorem III. Then in accordance with Theorem IV for each $b>G\left(R_{k}\right)\left(G\left(R_{k}\right)\right.$ is $R_{k}$-density of sequence $\left.\Lambda\right)$ there exists a sequence $\Gamma=\left\{\mu_{n}\right\}\left(0<\mu_{1} \leqslant \mu_{2} \leqslant \ldots \leqslant \mu_{n} \rightarrow \infty\right)$ contatining $\Lambda$ such that

$$
|M(t)-b t| \leqslant H(t) \quad(t \geqslant 0), \quad H \in R
$$

and entire function

$$
Q(z)=\prod_{n=1}^{\infty}\left(1-\frac{z^{2}}{\mu_{n}^{2}}\right) \quad(z=x+i y)
$$

of exponential type $\pi b$ possesses the properties

$1^{0} . Q\left(\lambda_{n}\right)=0, Q^{\prime}\left(\lambda_{n}\right) \neq 0(n \geqslant 1)$

$2^{0} \cdot \ln |Q(x)| \leqslant g(x)(x \geqslant 0), g \in L_{k}$;

$3^{0}$. The estimate

$$
\ln |Q(x)| \geqslant-C H(x) \ln ^{+} \frac{x}{H(x)}-2 \varphi(x)-D, \quad H \in R_{k}
$$

holds true as $x=r_{n}(n \geqslant 1)$. Estimates $2^{0}, 3^{0}$ in Theorem III follows from (13), (15). But since $H \in R_{k}, \varphi \in L_{k}$, there exists function $V \in L_{k}$ such that

$$
\ln |Q(z)| \geqslant \ln |Q(r)| \geqslant-V(r)
$$

as $r=r_{n}(r=|z|)(n \geqslant 1)$.

Let $\left\{r_{n}\right\}$ be the sequence from Theorem IV (estimates (19) hold true as $|z|=r_{n}(n \geqslant 1)$ ). Let $\Delta_{n}=\left(r_{p_{n}}, r_{p_{n}+1}\right)(n \geqslant 1)$ be all the intervals each of which contains at leas one point in $\Lambda$ (some of intervals $\left(r_{n}, r_{n+1}\right)$ can contain no points in $\Lambda$ ).

By $\Gamma_{p_{n}}(n \geqslant 1)$ we denote a closed contour formed by two arcs of the circles $K_{p_{n}}=\{\lambda:|\lambda|=$ $\left.r_{p_{n}}\right\}$ and $K_{p_{n}+1}=\left\{\lambda:|\lambda|=r_{p_{n}+1}\right\}$ in the angle $\left\{\lambda:|\arg \lambda| \leqslant \varphi_{n}<\frac{\pi}{4}\right\}$ and by the segments of the rays $\left\{\lambda:|\arg \lambda|=\varphi_{n}\right\}$.

In the proof of Theorem 1 we employ the functions

$$
q_{n}(\lambda)=\prod_{\nu_{k} \in \Delta_{n}}\left(1-\frac{\lambda}{\nu_{k}}\right)
$$

where $\Delta_{n}=\left(r_{p_{n}}, r_{p_{n}+1}\right), \nu=\left\{\nu_{k}\right\}=\Gamma \backslash \Lambda$. Sequence $\nu$ is constructed in the proof of Theorem IV and possesses the properties [6]:

a) $\inf _{i \neq j}\left|\nu_{i}-\nu_{j}\right| \geqslant \tau>0$

b) $\inf _{m \geqslant 1}\left|\lambda_{n}-\nu_{m}\right| \geqslant \frac{\gamma}{\varphi\left(2 \lambda_{n}\right)}(\gamma>0, n \geqslant 1)$, where $\varphi$ is the functions from condition (14) of Theorem IV. 
Let us establish estimates for $\left|q_{n}(\lambda)\right|$.

Lemma 1. There exists function $u \in L_{k}$ such that

$$
\max _{\lambda_{j} \in \Delta_{n}}|\ln | q_{n}\left(\lambda_{j}\right)|| \leqslant u\left(r_{p_{n}}\right) \quad(n \geqslant 1)
$$

Proof. Indeed, let $\lambda_{j} \in \Delta_{n}, \nu_{j}^{\prime}$ and $\nu_{j}^{\prime \prime}$ be the closest to $\lambda_{j}$ points of sequence $\nu$ located to the left and to right of $\lambda_{j}$, respectively. We have

$$
\left|\frac{\nu_{j}^{\prime}-\lambda_{j}}{\nu_{j}^{\prime}}\right|\left|\frac{\nu_{j}^{\prime \prime}-\lambda_{j}}{\nu_{j}^{\prime \prime}}\right| \geqslant\left[\frac{\gamma}{\varphi\left(2 \lambda_{j}\right)}\right]^{2} r_{p_{n}+1}^{-2} \quad\left(\lambda_{j} \in \Delta_{n}\right) .
$$

Since $1 \leqslant \varphi(x) \leqslant \alpha x \ln ^{+} x+\beta, r_{p_{n}} / r_{p_{n}+1} \rightarrow 1$ as $n \rightarrow \infty$, it implies the estimate

$$
\left|1-\frac{\lambda_{j}}{\nu_{j}^{\prime}}\right|\left|1-\frac{\lambda_{j}}{\nu_{j}^{\prime \prime}}\right| \geqslant e^{-c_{1}-c_{2} \ln r_{p_{n}}} \quad\left(\lambda_{j} \in \Delta_{n}\right),
$$

where $0<c_{i}<\infty(i=1,2)$.

Let $\Delta_{n}^{\prime}=\Delta_{n} \backslash\left\{\nu_{j}^{\prime}, \nu_{j}^{\prime \prime}\right\}$. Then

$$
\prod_{\substack{\nu_{k} \in \Delta_{n}^{\prime} \\ \nu_{k}<\lambda_{j}}}\left|\frac{\nu_{k}-\lambda_{j}}{\nu_{k}}\right| \geqslant\left(\frac{\tau}{r_{p_{n}+1}}\right)^{s_{n}} s_{n} !
$$

where $s_{n}$ is the amount of points $\nu_{k}<\lambda_{j}, \nu_{k} \in \Delta_{n}^{\prime}$. In the same way,

$$
\prod_{\substack{\nu_{k} \in \Delta_{n}^{\prime} \\ \nu_{k}>\lambda_{j}}}\left|\frac{\nu_{k}-\lambda_{j}}{\nu_{k}}\right| \geqslant\left(\frac{\tau}{r_{p_{n}+1}}\right)^{l_{n}} l_{n} !
$$

where $l_{n}$ is the amount of points $\nu_{k}>\lambda_{j}, \nu_{k} \in \Delta_{n}^{\prime}$. It follows from (21)-(23) that as $\lambda_{j} \in \Delta_{n}$, $(n \geqslant 1)$

$$
\left|q_{n}\left(\lambda_{j}\right)\right| \geqslant e^{-c_{1}-c_{2} \ln r_{p_{n}}}\left(\frac{\delta}{r_{p_{n}}}\right)^{s_{n}+l_{n}} s_{n} ! l_{n} ! \quad(0<\delta \leqslant 1) .
$$

If $\sup _{n \geqslant 1}\left(s_{n}+l_{n}\right)<\infty$, the required lower estimate for $\left|q_{n}\left(\lambda_{j}\right)\right|$ is obvious. Otherwise we first employ the known estimate

$$
s_{n} ! l_{n} ! \geqslant \frac{\left(s_{n}+l_{n}\right) !}{2^{s_{n}+l_{n}}}
$$

and then we use Stirling's asymptotic formula

$$
n ! \approx\left(\frac{n}{e}\right)^{n} \sqrt{2 \pi n}
$$

as $n \rightarrow \infty$.

Then by (24) we obtain

$$
\left|q_{n}\left(\lambda_{j}\right)\right| \geqslant \exp \left(-c_{3}-c_{2} \ln r_{p_{n}}\right)\left[\frac{\delta\left(s_{n}+l_{n}\right)}{2 e r_{p_{n}}}\right]^{s_{n}+l_{n}} \quad(n \geqslant 1),
$$

where $0<c_{i}<\infty(i=2,3)$. Letting $s_{n}+l_{n}=m_{n}$, for $\lambda_{j} \in \Delta_{n}$ we have

$$
\left|q_{n}\left(\lambda_{j}\right)\right| \geqslant \exp \left(-c_{3}-c_{2} \ln r_{p_{n}}-m_{n} \ln \frac{2 e r_{p_{n}}}{\delta m_{n}}\right)
$$

where $n \geqslant 1, m_{n}$ is a number not exceeding the amount of points $\nu_{k}$ in interval $\Delta_{n}$. Since $0<r_{p_{n}+1}-r_{p_{n}} \leqslant p H\left(p_{n}\right)(0<p<\infty)$, taking into consideration property a) of sequence $\nu$, we 
have $m_{n} \leqslant c_{4} H\left(r_{p_{n}}\right), 0<c_{4}<\infty(n \geqslant 1)$. Then $\frac{H(x)}{x} \downarrow 0$ as $x \uparrow \infty$ and function $\psi(x)=x \ln \frac{\Delta}{x}$ ( $\Delta$ is a positive constant) is increasing as $0<x<\frac{\Delta}{e}$. Therefore, by (25) we get that

$$
\ln \left|q_{n}\left(\lambda_{j}\right)\right| \geqslant-c_{5}-c_{2} \ln r_{p_{n}}-c_{6} H\left(r_{p_{n}}\right) \ln \frac{r_{p_{n}}}{H\left(r_{p_{n}}\right)}
$$

for $\lambda_{j} \in \Delta_{n}\left(n \geqslant n_{0}\right)$, where $0<c_{i}<\infty(i=2,5,6)$. Since $H \in R_{k}$, there exists $u_{1} \in L_{k}$ such that

for $\lambda_{j} \in \Delta_{n}$.

$$
\ln \left|q_{n}\left(\lambda_{j}\right)\right| \geqslant-u_{1}\left(r_{p_{n}}\right) \quad(n \geqslant 1)
$$

Let us estimate $\ln \left|q_{n}\left(\lambda_{j}\right)\right|$ from above. In order to do it, we observe that as $n \geqslant n_{1}$,

$$
\left|1-\frac{\lambda_{j}}{\nu_{k}}\right| \leqslant 1+\frac{r_{p_{n}+1}}{r_{p_{n}}} \leqslant e
$$

for each $\lambda_{j} \in \Delta_{n}$. Thus,

$$
\ln \left|q_{n}\left(\lambda_{j}\right)\right| \leqslant m_{n}+2 \leqslant c_{4} H\left(r_{p_{n}}\right)+2 \quad\left(n \geqslant n_{1}\right)
$$

for $\lambda_{j} \in \Delta_{n}$. It follows that

$$
\ln \left|q_{n}\left(\lambda_{j}\right)\right| \leqslant u_{2}\left(r_{p_{n}}\right) \quad(n \geqslant 1)
$$

for some function $u_{2} \in L_{k}$. Thus, by (26), (27) we finally obtain that

$$
\max _{\lambda_{j} \in \Delta_{n}}|\ln | q_{n}\left(\lambda_{j}\right)|| \leqslant u\left(r_{p_{n}}\right) \quad(n \geqslant 1),
$$

where $u=u_{1}+u_{2}$. The proof is complete.

We let $\gamma_{n}=\Gamma_{p_{n}}(n \geqslant 1)$.

Lemma 2. For each $n \geqslant 1$

$$
M_{n}=\max _{\lambda \in \gamma_{n}} \ln \left|q_{n}(\lambda)\right| \leqslant u\left(r_{p_{n}}\right),
$$

where $u$ is a function in $L_{k}$.

Proof. For each $\lambda \in \gamma_{n}, \nu_{k} \in \Delta_{n}$ as $n \geqslant n_{1}$ we have

$$
\left|1-\frac{\lambda}{\nu_{k}}\right| \leqslant 1+\frac{r_{p_{n}+1}}{r_{p_{n}}} \leqslant e .
$$

Therefore, as in Lemma $1, M_{n} \leqslant u_{2}\left(r_{p_{n}}\right) \leqslant u\left(r_{p_{n}}\right)(n \geqslant 1)$. Thus, estimate (28) indeed holds true.

Now we are in position to prove Theorem 1.

Proof of Theorem 1. Let $\gamma_{n}=\Gamma_{p_{n}}(n \geqslant 1)$. We let $\rho_{n}^{\prime}=r_{p_{n}}, \rho_{n}^{\prime \prime}=r_{p_{n}+1}$. Then $\Delta_{n}=\left(\rho_{n}^{\prime}, \rho_{n}^{\prime \prime}\right)$ $(n \geqslant 1)$.

We consider Dirichlet series

$$
F(s)=\sum_{j=1}^{\infty} a_{j} e^{\lambda_{j} s} \quad(s=\sigma+i t),
$$

where

$$
a_{j}=\exp \left(\left(\rho-q^{*}\right) \frac{\rho_{n}^{\prime}}{\ln _{k-1} \rho_{n}^{\prime}}\right) \frac{q_{n}\left(\lambda_{j}\right)}{Q^{\prime}\left(\lambda_{j}\right)} \quad(j \geqslant 1)
$$

for $\lambda_{j} \in \Delta_{n}(n \geqslant 1)$. Here $Q$ is function (18), $q_{n}$ is the function described in Lemmata 1,2 , $0 \leqslant \rho<\infty$, and $q^{*}$ is the quantity defined in Theorem III. Since $H \in R_{k}, \varphi \in L_{k}$, estimate (16) in Theorem IV implies that $q^{*}=q(Q) \geqslant 0$, where

$$
q(Q)=\varlimsup_{n \rightarrow \infty} \frac{\ln \lambda_{n}}{\lambda_{n}} \ln \left|\frac{1}{Q^{\prime}\left(\lambda_{n}\right)}\right| .
$$


Since $\rho_{n}^{\prime \prime} / \rho_{n}^{\prime} \rightarrow 1$ as $n \rightarrow \infty, q(Q)<\infty$, by (20) we obtain that

$$
\varlimsup_{j \rightarrow \infty} \frac{\ln \left|a_{j}\right|}{\lambda_{j}}=0 .
$$

Hence, $F \in D_{0}(\Lambda)$. Taking into consideration (20) once again and employing formula (11) for calculating $k$-order $\rho_{k}$, we have

$$
\begin{aligned}
\rho_{k}(F)= & \varlimsup_{j \rightarrow \infty} \frac{\ln _{k-1} \lambda_{j}}{\lambda_{j}} \ln _{k-1}\left|\frac{1}{Q^{\prime}\left(\lambda_{j}\right)}\right|+\lim _{j \rightarrow \infty} \frac{\ln _{k-1} \lambda_{j}}{\lambda_{j}} \ln \left|q_{n}\left(\lambda_{j}\right)\right| \\
& +\lim _{j \rightarrow \infty} \frac{\ln \lambda_{j}}{\lambda_{j}}\left(\rho-q^{*}\right) \frac{\rho_{n}^{\prime}}{\ln _{k-1} \rho_{n}^{\prime}}=q(Q)+\rho-q^{*}=\rho .
\end{aligned}
$$

Let us estimate order $\rho_{s}(F)$ in semi-strip $S\left(a, t_{0}\right)\left(a>\pi G\left(R_{k}\right)\right)$. Sequence $\Gamma=\left\{\mu_{n}\right\}$ of zeroes of function $Q$ has density $b$ that follows from $(17)$ and $G(R)<b$. Given $G\left(R_{k}\right)$ and $a$, we choose parameter $b$ in Theorem IV so that $G\left(R_{k}\right)<b<\frac{a}{\pi}$.

Then we observe that

$$
A_{n} \stackrel{\text { def }}{\equiv} \sum_{\lambda_{j} \in \Delta_{n}} a_{j} e^{\lambda_{j} s}=e^{\left(\rho-q^{*}\right) \frac{\rho_{n}^{\prime}}{\ln _{k-1} \rho_{n}^{\prime}}} \frac{1}{2 \pi i} \int_{\gamma_{n}} \frac{q_{n}(\xi)}{Q(\xi)} e^{s \xi} d \xi,
$$

where $\gamma_{n}$ is a closed contour formed by the $\operatorname{arcs}$ of circles $K_{\rho_{n}^{\prime}}$ and $K_{\rho_{n}^{\prime \prime}}$ in the angle $\{\lambda:|\arg \lambda| \leqslant$ $\left.\varphi_{n}<\frac{\pi}{4}\right\}$ and by the segments of rays $\left\{\lambda:|\arg \lambda|=\varphi_{n}\right\}$. We take $\varphi_{n}=\varepsilon_{0} \frac{H\left(\rho_{n}^{\prime}\right)}{\rho_{n}^{\prime}}\left(0<\varepsilon_{0}<1\right)$. Since $H \in R_{k}$, then $\varphi_{n} \downarrow 0$ as $n \rightarrow \infty$. We choose number $\varepsilon_{0}$ so that $0<\varphi_{n}<\frac{\pi}{4}(n \geqslant 1)$.

Let us estimate function $\left|\frac{q_{n}(\xi)}{Q(\xi)}\right|$ on contour $\gamma_{n}$. In order to do it, we employ (17) and estimate $[5]$

$$
-\ln \left|Q\left(r e^{ \pm i \varphi_{n}}\right)\right| \leqslant 6 H(r) \ln \frac{r}{H(r)}+\frac{8 \pi}{\left|\varphi_{n}\right|} \frac{H^{2}(r)}{r}+3 \mu_{1} b, \quad r \geqslant \rho_{n_{0}}^{\prime} .
$$

We note that this "effective" estimate of Weierstrass product is valid under the only restriction, which is condition (17).

Let $\rho_{n}^{\prime} \leqslant r \leqslant \rho_{n}^{\prime \prime}, n \geqslant n_{0}$. Since $\frac{H(r)}{r} \downarrow$ as $r \uparrow$, then $H(r) \leqslant \frac{r}{\rho_{n}^{\prime}} H\left(\rho_{n}^{\prime}\right) \leqslant \frac{\rho_{n}^{\prime \prime}}{\rho_{n}^{\prime}} H\left(\rho_{n}^{\prime}\right)$. Hence,

$$
-\ln \left|Q\left(r e^{ \pm i \varphi_{n}}\right)\right| \leqslant 12 H\left(\rho_{n}^{\prime}\right) \ln \frac{\rho_{n}^{\prime}}{H\left(\rho_{n}^{\prime}\right)}+\frac{32 \pi}{\varepsilon_{0}} H\left(\rho_{n}^{\prime}\right)+3 \mu_{1} b
$$

as $\lambda_{j} \in \Delta_{n}(n \geqslant 1)$. Estimates (19) hold true on the arcs of circles $K_{\rho_{n}^{\prime}}$ and $K_{\rho_{n}^{\prime \prime}}$ in contour $\gamma_{n}$. Since $H \in R_{k}$, in view of $\rho_{n}^{\prime \prime} / \rho_{n}^{\prime} \rightarrow 1$ as $n \rightarrow \infty$, by (19), (31) we obtain that

$$
-\ln |Q(\xi)| \leqslant w\left(\rho_{n}^{\prime}\right), \quad \xi \in \gamma_{n} \quad\left(n \geqslant n_{1}\right)
$$

for some function $w \in L_{k}$. Therefore, employing Lemma 2, we obtain the estimate

$$
\max _{\xi \in \gamma_{n}}\left|\frac{q_{n}(\xi)}{Q(\xi)}\right| \leqslant e^{u\left(\rho_{n}^{\prime}\right)+w\left(\rho_{n}^{\prime}\right)} \quad\left(n \geqslant n_{1}\right),
$$

where $u, w$ are functions in $L_{k}$. But then it follows from (30) that

$$
\left|A_{n}\right| \leqslant 2 \rho_{n}^{\prime \prime} e^{\left(\rho-q^{*}\right) \frac{\rho_{n}^{\prime}}{\ln \rho_{n}^{\prime}}+u\left(\rho_{n}^{\prime}\right)+w\left(\rho_{n}^{\prime}\right)} e^{\max _{\xi \in \gamma_{n}} \operatorname{Re}(s \xi)}
$$

as $n \geqslant n_{1}$.

Let $s \in S\left(a, t_{0}\right), \xi \in \gamma_{n}, s=\sigma+i t, \xi=\xi_{1}+i \xi_{2}$. Then

$$
\left|\sum_{\lambda_{j}<\rho_{n_{1}}^{\prime}} a_{j} e^{\lambda_{j} s}\right| \leqslant \sum_{\lambda_{j}<\rho_{n_{1}}^{\prime}}\left|a_{j}\right| e^{\lambda_{j} \sigma} \leqslant \sum_{\lambda_{j}<\rho_{n_{1}}^{\prime}}\left|a_{j}\right|=M,
$$


$\operatorname{Re}(s \xi)=\sigma \xi_{1}-t \xi_{2} \leqslant \sigma \rho_{n}^{\prime}+\left(\left|t_{0}\right|+a\right)|\operatorname{Im} \xi|$. Since $|\operatorname{Im} \xi| \leqslant \rho_{n}^{\prime \prime}\left|\sin \varphi_{n}\right| \leqslant \rho_{n}^{\prime}\left|\varphi_{n}\right|=\varepsilon_{0} \frac{\rho_{n}^{\prime \prime}}{\rho_{n}^{\prime}} H\left(\rho_{n}^{\prime}\right)$ as $\xi \in \gamma_{n}$, then there exists $d(0<d<\infty)$ such that

$$
\max _{\xi \in \gamma_{n}}(s \xi) \leqslant \sigma \rho_{n}^{\prime}+d H\left(\rho_{n}^{\prime}\right), \quad(n \geqslant 1)
$$

as $s \in S\left(a, t_{0}\right)$. Therefore, by (32)-(34) we obtain

$$
M_{s}(\sigma)=\max _{\left|t-t_{0}\right| \leqslant a}|F(\sigma+i t)| \leqslant M+\sum_{n=n_{1}}^{\infty} \gamma_{n} e^{\sigma \rho_{n}^{\prime}} \quad(\sigma<0),
$$

where

$$
\gamma_{n}=\exp \left[\ln \left(2 \rho_{n}^{\prime \prime}\right)+\left(\rho-q^{*}\right) \frac{\rho_{n}^{\prime}}{\ln \rho_{n}^{\prime}}+d H\left(\rho_{n}^{\prime}\right)+u\left(\rho_{n}^{\prime}\right)+w\left(\rho_{n}^{\prime}\right)\right] .
$$

We introduce an auxiliary series

$$
\Phi(s)=\sum_{n=1}^{\infty} \gamma_{n} e^{s \rho_{n}^{\prime}} \quad(s=\sigma+i t) .
$$

Since $H, u, w$ belong to $L_{k}, \rho_{n}^{\prime \prime} / \rho_{n}^{\prime} \rightarrow 1$ as $n \rightarrow \infty$, then in accordance with formula (11), the order of function $\Phi$ in half-plane $\Pi_{0}$ is equal to $\rho_{k}(\Phi)=\rho-q^{*}$. But $M_{s}(\sigma) \leqslant \Phi(\sigma)+M$. Hence, $\rho_{s}(F) \leqslant \rho-q^{*}$. It follows from Theorem III that $\rho_{k}(F) \leqslant \rho_{s}(F)+q^{*}$. Since $\rho_{k}(F)=\rho$, then $\rho_{k}(F)=\rho_{s}(F)+q^{*}$, and the proof of Theorem 1 is complete.

\section{BIBLIOGRAPHY}

1. J.F. Ritt. On certain points in the theory of Dirichlet series // Amer. J. Math. 50:1, 73-86 (1928).

2. S. Mandelbrojt. Séries adhérentes. Régularisation des suites. Applications. Gauthier-Villars, Paris (1952).

3. A.F. Leontiev. Exponential series. Nauka, Moscow (1976). (in Russian).

4. A.M. Gaŭsin. A bound for the growth in a half-strip of a function represented by a Dirichlet series // Matem. Sbornik. 117(159):3, 412-424 (1982). [Math. USSR-Sbornik. 43:3, 411-422 (1983).]

5. A.M. Gaisin, D.I. Sergeeva. Entire functions with a given sequence of zeros and of regular behavior on the real axis. I // Sibir. Matem. Zhurn. 48:5, 995-1007 (2007). [Siber. Math. J. 48:5, 798-808 (2007).]

6. A.M. Gaisin, D.I. Sergeeva. An estimate for the Dirichlet series in a half-strip in the case of the irregular distribution of exponents. II // Sibir. Matem. Zhurn. 49:2, 280-298 (2008). [Siber. Math. J. 49:2, 222-238 (2008).]

7. N.N. Aitkuzhina, A.M. Gaisin. Two-sided k-order estimate for Dirichlet series in a half-strip // Ufimskij Matem. Zhurn. 6:4, 19-31 (2014). [Ufa Math. J. 6:4, 18-30 (2014).]

Narkes Nurmukhametovna Aitkuzhina,

Bashkir State University,

Z. Validi str., 32,

450074, Ufa, Russia

E-mail: Yusupovan@rambler.ru

Akhtyar Magazovich Gaisin,

Institute of Mathematics CC USC RAS,

Chernyshevskii str., 112,

450008, Ufa, Russia

Bashkir State University,

Zaki Validi str., 32,

450074, Ufa, Russia

E-mail: Gaisinam@mail.ru 